\title{
RANDOM WALKS AND THE COLORED JONES FUNCTION
}

\author{
STAVROS GAROUFALIDIS AND MARTIN LOEBL
}

\begin{abstract}
It can be conjectured that the colored Jones function of a knot can be computed in terms of counting paths on the graph of a planar projection of a knot. On the combinatorial level, the colored Jones function can be replaced by its weight system. We give two curious formulas for the weight system of a colored Jones function: one in terms of the permanent of a matrix associated to a chord diagram, and another in terms of counting paths of intersecting chords.
\end{abstract}

\section{INTRODUCTION}

1.1. History. The colored Jones function of a knot is a 2-parameter formal power series $\sum_{n, m=0}^{\infty} a_{n, m} h^{n} \lambda^{m}$ which determines (and is determined by) the Jones polynomial of a knot and its cables, [BG]. The support of the colored Jones function lies in the triangle $0 \leq m \leq n$.

About 10 years ago, Melvin-Morton and Rozansky independently conjectured a relation among the diagonal terms $\sum_{n} a_{n, n}(h \lambda)^{n}$ of the colored Jones function of a knot and its Alexander polynomial, [MM, Ro1, Ro2]. D. Bar-Natan and the first author reduced the conjecture about knot invariants to a statement about their combinatorial weight systems, and then proved it for all semisimple Lie algebras using combinatorial methods, [BG].

Over the years, the MMR Conjecture has received attention by many researchers who gave alternative proofs, [Ch, KSA, KM, Ro3, V].

The subdiagonal terms $h^{k} \sum_{n} a_{n+k, n}(h \lambda)^{n}$ for a fixed $k$ of the colored Jones function, are (after a suitable parametrization) rational functions whose denominators are powers of the Alexander polynomial. This was first shown by Rozansky in [Ro3], who further conjectured that a similar property should be hold for the full Kontsevich integral of a knot. Rozansky's conjecture was recently settled by the first author and Kricker in [GK]. This opens the possibility of understanding each subdiagonal term of the colored Jones function (or the full Kontsevich integral) in topological terms. That said, not much is known about the subdiagonal terms of the colored Jones function. One can conjecture that each subdiagonal term is given in terms of a certain counting of random walks on a planar projection of a knot, see also Lin and Wang, [LW]. On a combinatorial level, the colored Jones function may be replaced by its weight system. In [BG] formulas for the weight system $W_{J}$ of the colored Jones function and of its leading order term $W_{J J}$ were given in terms of the intersection matrix of a chord diagram. In particular, $W_{J J}$ equals to the permanent of the intersection matrix of the chord diagram. In the last section of $[\mathrm{BG}]$ it was asked for a better understanding of the $W_{J}$ weight system, especially one that offers control over the subdiagonal terms in $W_{J}$.

The purpose of the paper is to give two curious combinatorial formulas for $W_{J}$ in Theorems 1 and 2 that answer these questions, and support the conjecture that the colored Jones polynomial is a counting of random walks.

1.2. Statement of the results. Consider the 0-framed colored Jones weight system

$$
W_{J}: \mathcal{A} \rightarrow \mathbb{Q}[\lambda]
$$

where $\mathcal{A}$ is the vector space over $\mathbb{Q}$ spanned by chord diagrams on an oriented line, modulo the 4 -term and 1-term relations, see $[\mathrm{BG}]$ and also below. We will normalize $W_{J}$ to equal 1 on the chord diagram with no

Date: This edition: February 28, $2002 \quad$ First edition: February 28, 2002.

S.G. was partially supported by an NSF and by an Israel-US BSF grant. M.L. was partly supported by GAUK 158 grant and by the Project LN00A056 of the Czech Ministry of Education. This and related preprints can also be obtained at http: //www.math.gatech.edu/ stavros 1991 Mathematics Classification. Primary 57N10. Secondary 57M25.

Key words and phrases: Colored Jones function, permanents, weight systems, random walks. 
chords (in [BG] the value of the empty chord diagram was $\lambda+1$ instead). With this normalization, it turns out that for a chord diagram $D, W_{J}(D)$ is a polynomial of $\lambda$ of degree the number of chords of $D . W_{J J}(D)$ is defined to be the coefficient of $\lambda^{\text {deg }}$ in $W_{J}$.

Given a chord diagram $D$, its chords are ordered (from left to right) and we can consider its intersection matrix $\operatorname{IM}(D)$ as in [BG, Definition 3.4] of size the number of chords of $D$ defined by

$$
\operatorname{IM}(D)_{i j}= \begin{cases}\operatorname{sign}(i-j) & \text { if the chords } i \text { and } j \text { of } D \text { intersect } \\ 0 & \text { otherwise. }\end{cases}
$$

We will consider a blown-up variant $\mathrm{IM}_{J}$ of the intersection matrix, of size 3 times the number of chords of $D$ composed of blocks of 3 by 3 matrices as follows:

$$
\operatorname{IM}_{J}(D)_{i j}= \begin{cases}A_{\operatorname{sign}(i-j)} & \text { if the distinct chords } i \text { and } j \text { of } D \text { intersect } \\ A_{0} & \text { if } i=j \\ A_{c} & \text { if chords } i, j \text { do not intersect and } i \text { is completely contained in } j \\ 0 & \text { otherwise, }\end{cases}
$$

where

$$
A_{0}=\left[\begin{array}{ccc}
\lambda+2 & 0 & 0 \\
0 & \lambda+2 & 1 \\
\lambda & -\lambda-2 & 1
\end{array}\right], \quad A_{-}=\left[\begin{array}{ccc}
0 & 0 & 0 \\
-1 & -1 & 0 \\
0 & 0 & 0
\end{array}\right], \quad A_{+}=\left[\begin{array}{lll}
1 & 1 & 0 \\
0 & 0 & 0 \\
0 & 0 & 0
\end{array}\right], \quad A_{c}=\left[\begin{array}{ccc}
1 & 1 & 0 \\
-1 & -1 & 0 \\
0 & 0 & 0
\end{array}\right]
$$

Example 1.1.

$$
D=\operatorname{IM}(D)=\left[\begin{array}{cccc}
0 & -1 & -1 & -1 \\
1 & 0 & 0 & -1 \\
1 & 0 & 0 & -1 \\
1 & 1 & 1 & 0
\end{array}\right], \quad \operatorname{IM}_{J}(D)=\left[\begin{array}{cccc}
A_{0} & A_{-} & A_{-} & A_{-} \\
A_{+} & A_{0} & 0 & A_{-} \\
A_{+} & A_{c} & A_{0} & A_{-} \\
A_{+} & A_{+} & A_{+} & A_{0}
\end{array}\right] .
$$

Theorem 1. We have

$$
W_{J}=\operatorname{Per}\left(\mathrm{IM}_{J}\right)
$$

where $\operatorname{Per}(A)$ denotes the permanent of a square matrix $A$.

There is an alternative (and equivalent) formula of $W_{J}$ in terms of counting cycles. In order to state it, given a chord diagram $D$ consider its labeled intersection graph $\operatorname{LIG}(D)$ as in [BG, Definition 3.4]. The vertices of $\operatorname{LIG}(D)$ correspond to the chords of $D$ (thus, are ordered) and the edges of $\operatorname{LIG}(D)$ correspond to the intersection of the chords of $D$.

We will use a variation $\operatorname{LID}(D)$, the labeled intersection digraph of $D$ defined as follows. Orient each edge from the smaller vertex to the larger and add an oriented loop on each vertex. The oriented loops are leaving the vertices. Next add directed edges $(i j)$ for each pair of chords $i, j$ such that $i$ is completely contained in $j$. In addition we color these new arcs red (and we draw them as $\quad r$ ) to distinguish them from the original arcs.

Example 1.2. For the chord diagram $D$ of Example 1.1, we have

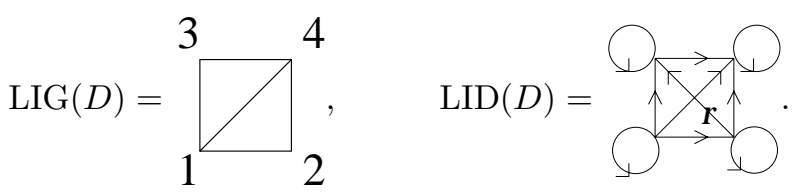

A bit more generally, consider a digraph $G=(V, A)$ (i.e., a directed graph) where $V$ is the set of vertices and $A$ is the set of arcs. If $e$ is an arc of $A$ with initial vertex $u$ and terminal vertex $v$ then we write $e=(u, v)$. We assume that there is one loop at each vertex and a loop at a vertex is considered as an arc leaving that vertex, and in addition some arcs which are not loops are red. We will consider the arcs with variables associated with them: the variable of an $\operatorname{arc} e$ is denoted by $x_{e}$. We will need the following notion of acceptable object, given $G$ :

Definition 1.3. A collection $K$ of arcs together with a thickening of one end of each of the $\operatorname{arcs}$ of $K$ is called acceptable for $G$ if the following properties are satisfied: 
- If $(i j)$ is a red arc of $G$ then both $\operatorname{arcs}(i j)$ and $(j i)$ may appear in $K$, but they must always be thickened at $i$. If $(i j)$ is an uncolored $\operatorname{arc}$ of $G$ then $(i j)$ with any end thickened may appear in $K$, but (ji) may not.

- Each vertex of $V$ is incident with 0,2 or 4 thickened $\operatorname{arcs}$ of $K$. If a loop belongs to $K$ then we assume it contributes 2 to the degree of the corresponding vertex. Moreover, a loop is always thickened at its initial segment, i.e., in agreement with its orientation.

- Exactly half of the arcs incident with a vertex are thickened at the vertex.

- If there are two arcs thickened at a vetex, then one of them enters and the other one leaves.

We will study the following partition function

$$
J(G)=\sum_{K \text { acceptable }} 2^{\operatorname{deg}_{4}(K)}(\lambda+2)^{|V|-\operatorname{deg}_{4}(K)} x_{K}(-1)^{a(K)}
$$

of a digraph $G$, where $x_{K}=\prod_{e \in K} x_{e}, \operatorname{deg}_{4}(K)$ denotes the number of vertices of $K$ incident with 4 arcs of $K$ and $a(K)$ is the number of arcs of $K$ with initial segment thickened, i.e., directed in agreement with the thickening.

The motivation for $J(G)$ comes from the case of the intersection digraph $\operatorname{LID}(D)$ of a chord diagram and the following:

Theorem 2. For a chord diagram $D$, we have

$$
W_{J}(D)=\left.J(\operatorname{LID}(D))\right|_{x_{e}=1} .
$$

Corollary 1.4. After a change of variables $d=\lambda+2$, let $W_{J J^{(n)}}$ denote the coefficient of $d^{\operatorname{deg}-n}$ in $W_{J}$. Then,

$$
W_{J J^{(n)}}(D)=2^{n} \sum_{K}(-1)^{a(K)}
$$

where the sum is over all acceptable $K$ such that $\operatorname{deg}_{4}(K)=n$.

Corollary 1.5. [BG] We have:

$$
W_{J J}=\operatorname{Per}(\mathrm{IM}) .
$$

How fast can one compute permanents?

Corollary 1.6. For general matrices of size $n$ we need $n !$ steps. However, a theorem of A. Galluccio [GL] and the second author implies that $W_{J}$ can be computed in $4^{g}$ steps, where $g$ is the genus of $\operatorname{LIG}(D)$, that is the smallest genus of a surface that LIG $(D)$ embeds.

1.3. Plan of the proof. In Section 2, we review the weight system of the colored Jones function, and reduce Theorem 2 to a Theorem 3 concerning digraphs. Section 3, is devoted to the proof of Theorem 3 using a trip to combinatorics. In Section 4, we translate our results using the language of permanents, and deduce Theorem 1. In the final Section 5 we prove the corollaries that follow Theorem 2.

1.4. Acknowledgement. The authors wish to thank the Georgia Institute of Technology which invited the second author and provided the environment of this research, and D. Bar-Natan for providing independent checks to the output of the program.

\section{Contents}

1. Introduction 1

1.1. History 1

1.2. Statement of the results 1

1.3. Plan of the proof 3

1.4. Acknowledgement 3

2. A review of the $W_{J}$ weight system 4

3. Understanding the state sums $J(G)$ and $J^{\prime}(G) \quad 6$

4. Converting to Permanents $\quad 9$

5. Proof of the corollaries 11

6. Numerical examples 12 
6.1. Running the program

6.2. The source code

\section{A REVIEW OF THE $W_{J}$ Weight SYSTEM}

The goal of this section is to reduce Theorem 2 to Theorem 3 stated below; this will be achieved by a careful examination of the $W_{J}$ weight system. Recall from [BG, Section 4.2] that $W_{J}$ can be computed as follows:

Step 1. Color each chord of a chord diagram $D$ by the following operator:

$$
\begin{aligned}
\hat{B}\left(v_{k} \otimes v_{k^{\prime}}\right) & =(k+1)\left(\lambda-k^{\prime}+1\right) v_{k+1} \otimes v_{k^{\prime}-1} \\
+ & (\lambda-k+1)\left(k^{\prime}+1\right) v_{k-1} \otimes v_{k^{\prime}+1} \\
+ & 1 / 2\left((\lambda-2 k)\left(\lambda-2 k^{\prime}\right)-\lambda(\lambda+2)\right) v_{k} \otimes v_{k^{\prime}}
\end{aligned}
$$

from [BG, p.121].

The key calculation is the following elementary rearrangement of $\hat{B}$, easily checked:

Lemma 2.1.

$$
\hat{B}\left(v_{k} \otimes v_{k^{\prime}}\right)=\left((\lambda+2) I+B^{+}+B^{-}\right)\left(v_{k} \otimes v_{k^{\prime}}\right)
$$

where

$$
\begin{array}{rlrl}
B^{+} & =\sum_{\epsilon=0,1}(-1)^{\epsilon} B_{\epsilon}^{+}, & B_{\epsilon}^{+} & =-(1+k)\left(\lambda+1-k^{\prime}\right) v_{k+\epsilon} \otimes v_{k^{\prime}-\epsilon} \\
B^{-} & =\sum_{\epsilon=0,1}(-1)^{\epsilon} B_{\epsilon}^{-}, & B_{\epsilon}^{-}=-\left(1+k^{\prime}\right)(\lambda+1-k) v_{k-\epsilon} \otimes v_{k^{\prime}+\epsilon} .
\end{array}
$$

This done, the coloring of chords of $D$ may be viewed as a function $\rho: \operatorname{chords}(D) \rightarrow\left\{I, B_{0}^{+}, B_{1}^{+}, B_{0}^{-}, B_{1}^{-}\right\}$.

Step 2. The end-points of the $n$ chords of $D$ partition the base line into $2 n+1$ segments $s_{0}, \ldots, s_{2 n}$ listed from left to right. We associate number $m\left(s_{i}\right)$ with each of these segments as follows:

1. Let $m\left(s_{0}\right)=0$.

2. If $i \geq 0$ and last point of $s_{i}$ is left end-vertex of chord $v$ then $m\left(s_{i+1}\right)$ is computed from $m\left(s_{i}\right)$ and $\rho(v)$ using 2.1:

- If $\rho(v) \in\left\{I, B_{0}^{+}, B_{0}^{-}\right\}$then $m\left(s_{i+1}\right)=m\left(s_{i}\right)$,

- If $\rho(v)=B_{1}^{+}$then $m\left(s_{i+1}\right)=m\left(s_{i}\right)+1$,

- If $\rho(v)=B_{1}^{-}$then $m\left(s_{i+1}\right)=m\left(s_{i}\right)-1$.

3. If $i \geq 0$ and last point of $s_{i}$ is right end-vertex of chord $v$ then $m\left(s_{i+1}\right)$ is computed from $m\left(s_{i}\right)$ and $\rho(v)$ using 2.1:

- If $\rho(v) \in\left\{I, B_{0}^{+}, B_{0}^{-}\right\}$then $m\left(s_{i+1}\right)=m\left(s_{i}\right)$,

- If $\rho(v)=B_{1}^{+}$then $m\left(s_{i+1}\right)=m\left(s_{i}\right)-1$,

- If $\rho(v)=B_{1}^{-}$then $m\left(s_{i+1}\right)=m\left(s_{i}\right)+1$.

Step 3. We let

$$
W_{J}(D)=\sum_{\rho} \prod_{\text {chords } v} \omega_{\rho}(v)
$$

where $\rho$ is a coloring of the chords of $D$ and $\omega_{\rho}(v)$ is a specific weight that is computed using Lemma 2.1 again:

- $\omega_{\rho}(v)=\lambda+2$ if $\rho(v)=I$,

- $\omega_{\rho}(v)=-(-1)^{\epsilon}(1+k)\left(\lambda+1-k^{\prime}\right)$ if $\rho(v)=B_{\epsilon}^{+}$,

- $\omega_{\rho}(v)=-(-1)^{\epsilon}\left(1+k^{\prime}\right)(\lambda+1-k)$ if $\rho(v)=B_{\epsilon}^{-}$,

where $k=m\left(s_{L}(v)\right), k^{\prime}=m\left(s_{R}(v)\right), s_{L}(v)$ is the segment ending at the left end-point of $v$ and $s_{R}(v)$ is the segment ending at the right end-point of $v$.

The reader is urged to look at [BG, Chapter 4] for an explanation of the above algorithm in terms of the representation theory of the $\mathfrak{s l}_{2}$ Lie algebra. 
Example 2.2. For the following coloring of the chord diagram of Example 1.1

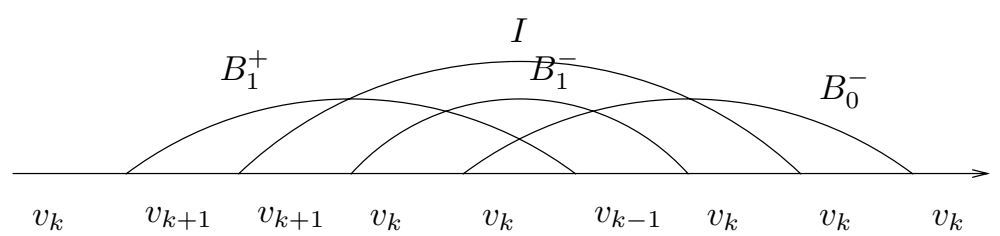

we have (assuming $m\left(s_{0}\right)=k$ ) that

$$
\begin{array}{ll}
\omega_{\rho}(1)=(1+k)(\lambda+1-k), & \omega_{\rho}(2)=\lambda+2 \\
\omega_{\rho}(3)=(1+(k-1))(\lambda+1-(k+1)), & \omega_{\rho}(4)=-(1+k)(\lambda+1-k) .
\end{array}
$$

Each coloring $\rho$ of the chords is determined by a subset $V^{\prime}$ of chords such that $\rho(v)=I$ for $v \notin V^{\prime}$ and by a coloring $c$ where $c(v)$ is an assignement of an element $\left(\epsilon_{v}, \delta_{v}\right) \in\{0,1\} \times\{+,-\}$ for each $v \in V^{\prime}$. Hence we can write

$$
W_{J}(D)=\left.J^{\prime}(\operatorname{LID}(D))\right|_{x_{e}=1}
$$

where

$$
J^{\prime}(G)=\sum_{V^{\prime} \subset V}(\lambda+2)^{\left|V-V^{\prime}\right|} \sum_{c \text { col of }} \prod_{V^{\prime}} \omega_{v \in V^{\prime}} \omega_{c}^{\prime}(v) .
$$

An important observation is that $\omega_{c}^{\prime}(v)$ can be computed in terms of the local structure of the labeled intersection digraph $\operatorname{LID}(D)$. The next lemma describes this.

Lemma 2.3. Let $\operatorname{LID}(D)=(V, A)$. Then

$$
\omega_{c}^{\prime}(v)=-(-1)^{\epsilon_{v}}\left(1+\sum_{e \in A, v \in e} z_{v}(e)\right)\left(\lambda+1-\sum_{e \in A, v \in e} \bar{z}_{v}(e)\right)
$$

where $z_{v}, \bar{z}_{v}$ are defined as follows:

- If e uncolored then

- If $c(v)=(+, \epsilon)$ and $e=(w, v)$ then $z_{v}(e)=\delta_{w} \epsilon_{w} x_{e}$,

- If $c(v)=(+, \epsilon)$ and $e=(v, w)$ then $\bar{z}_{v}(e)=\delta_{w} \epsilon_{w} x_{e}$,

- If $c(v)=(-, \epsilon)$ and $e=(w, v)$ then $\bar{z}_{v}(e)=\delta_{w} \epsilon_{w} x_{e}$,

- If $c(v)=(-, \epsilon)$ and $e=(v, w)$ then $z_{v}(e)=\delta_{w} \epsilon_{w} x_{e}$,

- If $e$ is red and $e=(v, w)$ then $z_{v}(e)=\bar{z}_{v}(e)=\delta_{w} \epsilon_{w} x_{e}$,

- and $z_{v}(e)=\bar{z}_{v}(e)=0$ otherwise.

Proof. If $c(v)=(+, \epsilon)$ then $c(v)$ corresponds to the operator $B_{\epsilon}^{+}$and hence $\left.\omega_{c}^{\prime}(v)\right|_{x_{e}=1}=-(-1)^{\epsilon_{v}}(1+k)(\lambda+$ $\left.1-k^{\prime}\right)$. A moment's thought reveals that $k=k_{1}-k_{2}+k_{3}-k_{4}$ and $k^{\prime}=k_{1}^{\prime}-k_{2}^{\prime}+k_{3}-k_{4}$ where

$$
\begin{array}{rlrl}
k_{1} & =\mid\{e=(w, v) \text { uncolored; } c(w)=(+, 1)\} \mid, & & k_{2}=\mid\{e=(w, v) \text { uncolored; } c(w)=(-, 1)\} \mid \\
k_{1}^{\prime} & =\mid\{e=(v, w) \text { uncolored; } c(w)=(+, 1)\} \mid, & k_{2}^{\prime}=\mid\{e=(v, w) \text { uncolored; } c(w)=(-, 1)\} \mid \\
k_{3}=\mid\{e=(v, w) \text { red; } c(w)=(+, 1)\} \mid, & k_{4}=\mid\{e=(v, w) \text { red; } c(w)=(-, 1)\} \mid .
\end{array}
$$

The reasoning is analogous for $c(v)=(-, \epsilon)$.

Thus, Theorem 2 follows from the following

Theorem 3. For all digraphs $G$ with one loop at each vertex, we have

$$
J^{\prime}(G)=J(G) .
$$




\section{Understanding the state sums $J(G)$ And $J^{\prime}(G)$}

In this section we prove Theorem 3, via a trip to combinatorics with curious cancellations caused by applications of the binomial theorem.

Let us begin by rewriting $J^{\prime}(G)$. Let $\epsilon_{V^{\prime}}=\sum_{v \in V^{\prime}} \epsilon_{v}$. Then,

$$
\begin{aligned}
J^{\prime}(G)= & \sum_{V^{\prime} \subset V}(-1)^{\left|V^{\prime}\right|}(\lambda+2)^{\left|V-V^{\prime}\right|} \\
& \sum_{c \text { col of } V^{\prime}}(-1)^{\epsilon_{V^{\prime}}} \sum_{V_{1} \subset V^{\prime}, V_{2} \subset V^{\prime}}(-1)^{\left|V_{2}\right|}(\lambda+1)^{\left|V^{\prime}-V_{2}\right|} \prod_{v \in V_{1}}\left(\sum_{v \in e} z_{v}(e)\right) \prod_{v \in V_{2}}\left(\sum_{v \in e} \bar{z}_{v}(e)\right)
\end{aligned}
$$

where $V_{1}$ and $V_{2}$ are possibly overlapping subsets of $V$.

Note that

$$
\prod_{v \in V_{1}}\left(\sum_{v \in e} z_{v}(e)\right)=\sum_{f} \prod_{v \in V_{1}} z_{v}(e(f, v))
$$

where $f: V_{1} \rightarrow A$ maps $v$ to the arc denoted by $e(f, v)$ such that $v \in e(f, v)$ and moreover if $e(f, v)$ red then $e=(v,$.$) , i.e. e$ starts in $v$. In other words, $f$ associates with each vertex $v$ of $V_{1}$ an arc incident with it. Similarly, we can rewrite $\prod_{v \in V_{2}}\left(\sum_{v \in e} \bar{z}_{v}(e)\right)$. Hence,

$$
\begin{aligned}
J^{\prime}(G)= & \sum_{V^{\prime} \subset V, V_{1} \subset V^{\prime}, V_{2} \subset V^{\prime}}(-1)^{\left|V^{\prime}\right|}(\lambda+2)^{\left|V-V^{\prime}\right|}(\lambda+1)^{\left|V^{\prime}-V_{2}\right|}(-1)^{\left|V_{2}\right|} \\
& \sum_{f: V_{1} \rightarrow A, g: V_{2} \rightarrow A c \text { col of } V^{\prime}}(-1)^{\epsilon_{V^{\prime}}} \prod_{v \in V_{1}}\left(z_{v}(e(f, v))\right) \prod_{v \in V_{2}}\left(\bar{z}_{v}(e(g, v))\right) .
\end{aligned}
$$

Let us rewrite the formula more: we fix $W_{1}=V_{1} \cap V_{2}, W_{2}=V_{1} \cup V_{2}$ and we let $h$ to be disjoint union of $f$ and $g$.

Remark 3.1. What exactly is $h$ ? Answer: $h$ is a function that assigns to each vertex of $V^{\prime}$ zero, one or two arcs incident with it (if the arc is red then it must start in that vertex). Hence $|h(v)| \leq 2$ for all $v \in V^{\prime}$ and if $e \in h(v)$ then $v \in e$. Here we slowly move towards the formalism of acceptable objects. If $e \in h(v)$ then thicken the end of $e$ containing $v$. Hence $h$ becomes a system of thickened $\operatorname{arcs}$ of $G$ so that there are at most two arcs in the system that are thickened at each vertex of $V^{\prime}$, and red arcs are thickened always at the start.

If we have such an $h$, then $W_{1}=\{v:|h(v)|=2\}$ and $W_{2}=\{v:|h(v)| \geq 1\}$. Hence, $h$ determines the sets $W_{1}$ and $W_{2}$. Fix an $h$ as above, consider its corresponding sets $W_{1}, W_{2}$, and let $h\left(W_{2}\right)$ denote the system of thickened arcs determined by $h$. We have

$$
J^{\prime}(G)=\sum_{V^{\prime} \subset V}(-1)^{\left|V^{\prime}\right|}(\lambda+2)^{\left|V-V^{\prime}\right|} \sum_{h} A\left(V^{\prime}, h\right)
$$

where

$$
A\left(V^{\prime}, h\right)=\sum_{c \text { col of } V^{\prime}} \sum_{V_{2}^{\prime} \subset W_{2}-W_{1}} \sum_{g: W_{1} \cup V_{2}^{\prime} \rightarrow h\left(W_{2}\right): g(v) \in h(v)} B
$$

and

$$
B=(-1)^{\epsilon_{V^{\prime}}}(-1)^{\left|V_{2}^{\prime} \cup W_{1}\right|}(\lambda+1)^{\left|V^{\prime}-\left(W_{1} \cup V_{2}^{\prime}\right)\right|} \prod_{v \in W_{1} \cup V_{2}^{\prime}} \bar{z}_{v}(e(g, v)) \prod_{v \in W_{1} \cup V_{1}^{\prime}} z_{v}(e(f, v))
$$

where $V_{1}^{\prime}=W_{2}-\left(W_{1} \cup V_{2}^{\prime}\right)$ and $f: W_{1} \cup V_{1}^{\prime} \rightarrow h\left(W_{2}\right)$ is such that the disjoint union of $f$ and $g$ is $h$.

The next two lemmas restrict the possible configurations of $h$ that contribute non-zero $A\left(V^{\prime}, h\right)$.

Lemma 3.2. Let $v \in W_{2}$. If the only arcs of $h\left(W_{2}\right)$ incident with $v$ are the arcs of $h(v)$, then $A\left(V^{\prime}, h\right)=0$.

Proof.

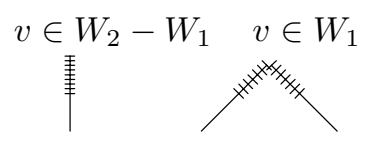


Fix $V_{2}^{\prime}, g$ and $c\left(V^{\prime}-\{v\}\right)$. If $B \neq 0$, then the color $\delta_{v} \in\{-,+\}$ of $v$ may be determined by $g$ and the

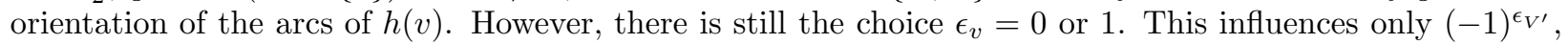
hence the lemma follows.

Lemma 3.3. Let $v \in W_{1}$. If both arcs of $h(v)$ are uncolored and oriented in the same way w.r.t. $v$ then $A\left(V^{\prime}, h\right)=0$. If there are exactly three arcs of $h\left(W_{2}\right)$ incident with $v$ then $A\left(V^{\prime}, h\right)=0$.

Proof.

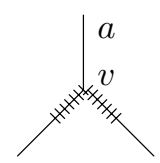

Since $v \in W_{1}$ we have $|h(v)|=2$. For the first part, if both $\operatorname{arcs}$ of $h(v)$ are uncolored and oriented in the same way, then there is no way to choose color $\delta_{v} \in\{-,+\}$ so that $B \neq 0$. For the second part, if there are exactly three $\operatorname{arcs}$ of $h\left(W_{2}\right)$ incident with $v$ then there is exactly one arc, say $a$, which is incident with $v$ and belongs to $h\left(W_{2}\right)-h(v)$. Fix $V_{2}^{\prime}$ and consider pairs $g_{1}, g_{2}$ of functions $g$ which differ only on $v$. If $B \neq 0$ and at least one of the $\operatorname{arcs}$ of $h(v)$ is uncolored, then the color $\delta_{v} \in\{-,+\}$ of $v$ is determined by the orientation of the $\operatorname{arcs}$ of $h(v)$ and the choice between $g_{1}$ and $g_{2}$. This color is opposite for $g_{1}$ and $g_{2}$, and so the edge $a$ is counted with different signs for $g_{1}$ and $g_{2}$ while all the rest remains the same, hence the total contribution is 0 . If both arcs of $h(v)$ are red then both colors $\delta_{v} \in\{-,+\}$ of $v$ are possible for $B \neq 0$ and both $g_{1}$ and $g_{2}$. Hence again the edge $a$ contributes twice +1 and twice -1 and the total contribution is 0 .

Note that the second property of the above lemma assures that the system $h\left(W_{2}\right)$ of thickened uncolored edges is a set for each $h$ which contributes a non-zero term to $J^{\prime}(G)$.

Corollary 3.4. (a) If e $\in h\left(W_{2}\right)$, then both vertices of e belong to $W_{2}$.

(b) Each vertex of $W_{2}$ has degree (i.e., valency) 2 or 4 in $h\left(W_{2}\right)$ and if $N(v)$ is the set of edges of $h\left(W_{2}\right)$ incident with $v$ then $|N(v)|=2|h(v)|$. In other words, the allowed configurations are

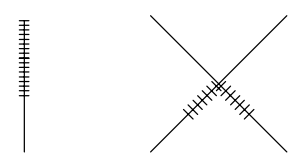

Proof. It follows from Lemmas 3.2 and 3.3 that $|N(v)| \geq 2|h(v)|$ at each vertex $v$. On the other hand, each edge of $h\left(W_{2}\right)$ has one thick end and one thin end, and so there cannot be more thin ends than thick ends. Hence $|N(v)|=2|h(v)|$ and the corollary follows.

Corollary 3.5. If $W_{2} \neq V^{\prime}$, then $A\left(V^{\prime}, h\right)=0$.

Proof. We write

$$
A\left(V^{\prime}, h\right)=\sum_{c \text { col of } V^{\prime}-W_{2}}(-1)^{\epsilon_{V^{\prime}-W_{2}}} \text { (rest) }
$$

where the 'rest' is not influenced by the colorings in $V^{\prime}-W_{2}$. Hence,

$$
A\left(V^{\prime}, h\right)=(\text { rest }) \sum_{C \subset V^{\prime}-W_{2}}(-1)^{|C|} 2^{\left|V^{\prime}-W_{2}\right|}
$$

which vanishes unless $V^{\prime}=W_{2}$.

Summarizing, a function $h$ such that $A\left(V^{\prime}, h\right) \neq 0$ determines a collection of thickened arcs that is almost an acceptable object:

- each vertex has degree 2 or 4 in $V^{\prime}$ and 0 in $V-V^{\prime}$,

- exactly half of the arcs incident with a vertex are thickened at that vertex,

- if there are two uncolored arcs thickened at a vertex then they have opposite orientation with respect to the vertex,

- the red arcs are always thickened at the start. 
Let us call such object good on $V^{\prime}$. Note also that for each coloring of a good object on $V^{\prime}$ which contributes non-zero to $B$ we must have $\epsilon_{v}=1$ for each $v \in V^{\prime}$ and hence $(-1)^{\epsilon} V^{\prime}=(-1)^{\left|V^{\prime}\right|}$. Collecting our efforts so far, we have

$$
J^{\prime}(G)=\sum_{V^{\prime} \subset V}(\lambda+2)^{\left|V-V^{\prime}\right|} \sum_{K \text { good on } V^{\prime}} A^{\prime}\left(V^{\prime}, K\right)
$$

where

$$
A^{\prime}\left(V^{\prime}, K\right)=\sum_{V_{2}^{\prime} \subset V^{\prime}-W_{1}}(\lambda+1)^{\left|V^{\prime}-\left(W_{1} \cup V_{2}^{\prime}\right)\right|} C\left(V_{2}^{\prime}, V^{\prime}, K\right),
$$

$W_{1}$ is the set of vertices of $V^{\prime}$ of degree 4 in $K$ and

$$
C\left(V_{2}^{\prime}, V^{\prime}, K\right)=\sum_{g^{\prime}: W_{1} \rightarrow K}(-1)^{\left|W_{1}\right|}(-1)^{\left|V_{2}^{\prime}\right|} \sum_{c \text { col }} \prod_{v \in W_{1} \cup V_{2}^{\prime}} \bar{z}_{v}(e(g, v)) \prod_{v \in V^{\prime}-V_{2}^{\prime}} z_{v}(e(f, v)),
$$

and $g^{\prime}, g, f$ have the following properties:

- if $p \in\left\{g^{\prime}, g, f\right\}$ then $p(v)$ is an arc of $K$ incident with $v$ and thickened at $v$,

- $g: W_{1} \cup V_{2}^{\prime} \rightarrow K$ is unique such function extending $g^{\prime}$,

- $f: V^{\prime}-V_{2}^{\prime} \rightarrow K$ is unique such function with $f \cup g=K$.

Lemma 3.6. $C\left(X, V^{\prime}, K\right)=C\left(Y, V^{\prime}, K\right)$ for arbitrary $X, Y$ subsets of $V^{\prime}-W_{1}$.

Proof. Exactly half of the edges of $K$ incident with each vertex are thickened at that vertex and hence $K$ may be regarded as a union of cycles $Z_{1}, \ldots, Z_{m}$ such that each $Z_{i}$ has the form

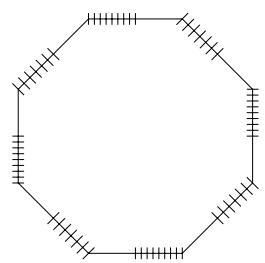

and such that each vertex of $K$ lies in at most two of the cycles $Z_{i}$. In other words, we may think that $K$ is pictured schematically as follows

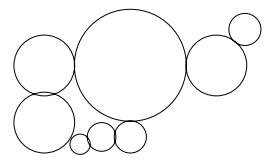

(where for simplicity, we have drawn the cycles $Z_{i}$ as circles). As we observed above,

$$
C\left(X, V^{\prime}, K\right)=\sum_{g^{\prime}: W_{1} \rightarrow K}(-1)^{\left|W_{1}\right|}(-1)^{|X|} \sum_{c \text { col }} \prod_{v \in W_{1} \cup X} \bar{z}_{v}(e(g, v)) \prod_{v \in V^{\prime}-X} z_{v}(e(f, v))
$$

It suffices to show the following

\section{Claim.}

$$
C\left(X, V^{\prime}, K\right)=\sum_{K^{\prime}}(-1)^{a\left(K^{\prime}\right)} x_{K}
$$

where $K^{\prime}$ is any collection of thickened arcs obtained from $K$ by changing orientation of some (possibly none) red $\operatorname{arcs}$ of $K$ so that $K^{\prime}$ is an acceptable object (i.e. if two arcs are thickened at a vertex then they are oppositely oriented w.r.t. that vertex), and $a\left(K^{\prime}\right)$ is the number of arcs of $K^{\prime}$ directed in agreement with the thickening.

Proof of the Claim. We can write

$$
C\left(X, V^{\prime}, K\right)=\sum_{g^{\prime}: W_{1} \rightarrow K} D
$$

and

$$
D=(-1)^{\left|W_{1}\right|}(-1)^{|X|} \sum_{c \text { col }} \prod_{v \in W_{1} \cup X} \bar{z}_{v}(e(g, v)) \prod_{v \in V^{\prime}-X} z_{v}(e(f, v)) .
$$

First we observe that the Claim is true when $K$ has a vertex of degree 2 where the thickened edge is red. Indeed, in this case both sides of the formula in the Claim equal 0. Hence let $K$ donot have such a vertex. 
Let $S$ be the set of vertices of $K$ where two red arcs are thickened. Observe that exactly $2^{|S|}$ colorings $c$ contribute a non-zero term to $D$ : we observed before that necessarily $\epsilon(v)=1$ for each $v \in V^{\prime}$. Moreover, each color $\delta(v)$ contributing non-zero to $D$ is uniquely determined for each $v$ where at least one uncolored arc is thickened: explicitly, let $v$ be a vertex of $Z_{i}$ and let $e$ be the unique arc of $Z_{i}$ thickened at $v$, and let $e$ be uncolored. Then $\delta(v)$ depends on the orientation of $e$ and whether $e$ belongs to $g$ or not. Hence there is at most one coloring $\delta(v)$ which contributes a non-zero term to $D$. Also observe that the unique 'non-zero coloring' of these vertices of each $Z_{i}$ compose well together: this follows from the fact that if $v$ is a vertex of degree 4 in $K$ then exactly 2 arcs are thickened at $v$, and if both are uncolored then they have different orientation with respect to $v$ and one belongs to $g$ and the other belongs to $f$. Finally observe that for vertex $v$ where two red arcs are thickened, any $\delta(v)$ contributes a non-zero.

Next we will observe that the contribution of each of these $2^{|S|}$ colorings to $D$ is the same and equals $(-1)^{a\left(K^{\prime}\right)} x_{K}$ where $K^{\prime}$ is any collection of thickened arcs obtained from $K$ by changing orientation of some (possibly none) red arcs of $K$ so that $K^{\prime}$ is an acceptable object (i.e., if two arcs are thickened at a vertex then they are opositely oriented with respect to that vertex). This proves the Claim since the number of objects $K^{\prime}$ equals $2^{|S|}$. Hence it remains to confirm the contribution of each of the allowed colorings to $D$.

First observe that it is true when $X=\emptyset$. Next, let us put a vertex $v$ of $Z_{i}-W_{1}$ into $X$ and let $e$ be the arc of $Z_{i}$ thickened at $v$. Then $e$ is uncolored by our assumption and we need to change $\delta(v)$ in order to have a nonzero contribution, hence the product of signs along $Z_{i}$ changes but $(-1)^{|X|}$ also changes and so the final total sign is the same.

We let

$$
\begin{aligned}
C\left(V^{\prime}, K\right) & =\sum_{g^{\prime}: W_{1} \rightarrow K} \sum_{K^{\prime}}(-1)^{a\left(K^{\prime}\right)} x_{K} \\
& =2^{\operatorname{deg}_{4}(K)} \sum_{K^{\prime}}(-1)^{a\left(K^{\prime}\right)} x_{K} .
\end{aligned}
$$

Equation (1) together with Lemma 3.6 implies that

$$
\begin{aligned}
J^{\prime}(G) & =\sum_{V^{\prime} \subset V}(\lambda+2)^{\left|V-V^{\prime}\right|} \sum_{K \text { good on } V^{\prime}} C\left(V^{\prime}, K\right) \sum_{A \subset V^{\prime}-W_{1}}(\lambda+1)^{|A|} \\
& =\sum_{V^{\prime} \subset V}(\lambda+2)^{\left|V-V^{\prime}\right|} \sum_{K \text { good on } V^{\prime}} C\left(V^{\prime}, K\right)(\lambda+2)^{\left|V^{\prime}-W_{1}\right|} \\
& =\sum_{K \text { good on } V^{\prime}}(\lambda+2)^{\left|V-W_{1}\right|} C\left(V^{\prime}, K\right) \\
& =\sum_{K \text { acceptable }}(\lambda+2)^{|V|-\operatorname{deg}_{4}(K)} 2^{\operatorname{deg}_{4}(K)}(-1)^{a(K)} x_{K} \\
& =J(G)
\end{aligned}
$$

which concludes the proof of Theorem 3.

\section{Converting to Permanents}

The goal of this section is to convert the state sum $J(D)$ into a permanent, in the following way:

\section{Theorem 4.}

$$
\left.J(\operatorname{LID}(D))\right|_{x_{e}=1}=\operatorname{Per}\left(\operatorname{IM}_{J}(D)\right)
$$

Note that Theorem 1 follows from Theorems 2 and 4.

We will achieve the conversion of $J(\operatorname{LID}(D))$ in a permanent by a local modification (we can say, a blowup) of each of the vertices of the original digraph $\operatorname{LID}(D)$. It turns out that the modification triples each of the vertices of $G$. Why triple? Because in a sense $J(\operatorname{LID}(D))$ has to do with the $\mathfrak{s l}_{2}$-Lie algebra. Thus, we are back to Lie algebras, this time through a common blow-up trick of combinatorics. 
Proof (of Theorem 4). We have that

$$
\left.J(\operatorname{LID}(D))\right|_{x_{e}=1}=\sum_{K \text { acceptable }}(\lambda+2)^{|V|-\operatorname{deg}_{4}(K)} 2^{\operatorname{deg}_{4}(K)}(-1)^{a(K)},
$$

where $a(K)$ equals the number of arcs of $K$ thickened in agreement with their orientation. A single loop of $K$ is always leaving its vertex, and it is directed in agreement with its thickening. Hence each single loop contributes ' $(-1)$ ' to $a(K)$. Let us now get rid of these single loops: an acceptable object without single loops will be called connected and a connected object where each vertex of $V$ has degree at least 2 will be super. We have

$$
\begin{aligned}
\left.J(\operatorname{LID}(D))\right|_{x_{e}=1} & =\sum_{K \text { acceptable }}(\lambda+2)^{|V|-\operatorname{deg}_{4}(K)} 2^{\operatorname{deg}_{4}(K)}(-1)^{a(K)} \\
& =\sum_{K \text { connected }}(\lambda+2)^{|V|-\operatorname{deg}_{4}(K)} 2^{\operatorname{deg}_{4}(K)}(-1)^{a(K)} \sum_{U \subset V-K}(-1)^{|U|} \\
& =\sum_{K \text { super }}(\lambda+2)^{|V|-\operatorname{deg}_{4}(K)} 2^{\operatorname{deg}_{4}(K)}(-1)^{a(K)} .
\end{aligned}
$$

We will use a variation $\operatorname{TID}(D)$ ), the thickened intersection digraph of $D$ defined as follows. Double each of the arcs of $\operatorname{LID}(D)$ and:

1. if the arc is uncolored then thicken such pair at opposite ends,

2. if the arc is red then thicken each arc of the pair at the start, and then change the orientation of one of them,

3. thicken each loop at its initial segment, i.e., in agreement with its orientation.

In pictures, the thickening of LID is the substitution
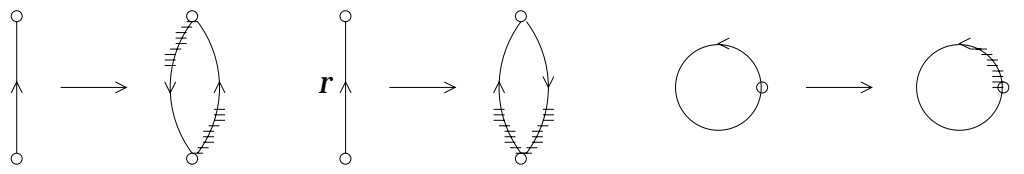

Now we can write

$$
\left.J(\operatorname{LID}(D))\right|_{x_{e}=1}=\sum_{K \text { super subobject of } \operatorname{TID}(D)}(\lambda+2)^{|V|-\operatorname{deg}_{4}(K)} 2^{\operatorname{deg}_{4}(K)}(-1)^{a(K)} .
$$

Let us describe now how a thickened digraph $D\left(\operatorname{IM}_{J}\right)$ may be constructed from $\operatorname{TID}(D)$. The construction easily follows from the definition of matrix $\operatorname{IM}_{J}$ : it consists in replacing each vertex of $\operatorname{TID}(D)$ by a 'gadget' on three vertices, as follows:

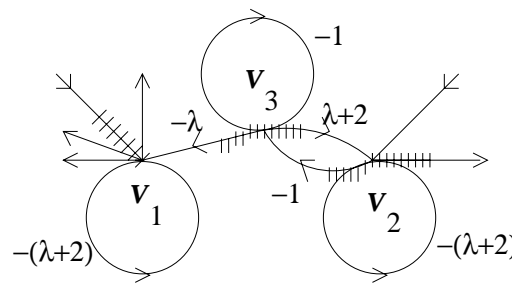

The construction goes as follows:

1. For each vertex $v$ of $\operatorname{TID}(D)$ introduce three vertices $v_{1}, v_{2}, v_{3}$ for $D\left(\operatorname{IM}_{J}\right)$.

2. Define the thickened arcs and their weights among each triple $v_{1}, v_{2}, v_{3}$ as follows:

- add loop $l_{i}$ at each $v_{i}$ and let $w\left(l_{1}\right)=w\left(l_{2}\right)=-(\lambda+2)$ and $w\left(l_{3}\right)=-1$,

- add $\operatorname{arc}\left(v_{3}, v_{1}\right)$ thickened at $v_{3}$ with weight $-\lambda$,

- add arc $\left(v_{3}, v_{2}\right)$ thickened at $v_{3}$ with weight $\lambda+2$,

- add arc $\left(v_{2}, v_{3}\right)$ thickened at $v_{2}$ with weight -1 .

3. For each thickened $\operatorname{arc}(u, w)$ of $\operatorname{TID}(D)$ do the following:

- If $(u, w)$ is thickened at $u$ then add $\left(u_{2}, w_{1}\right),\left(u_{2}, w_{2}\right)$ thickened at $u_{2}$ with weights equal to 1 ,

- If $(u, w)$ is thickened at $w$ then add $\left(u_{1}, w_{1}\right),\left(u_{2}, w_{1}\right)$ thickened at $w_{1}$ with weights equal to 1 . 
It follows directly from the definition of the permanent that

$$
\operatorname{Per}\left(\mathrm{IM}_{J}\right)=\sum_{L \in \mathcal{L}}(-1)^{a(L)} w_{L}
$$

where $\mathcal{L}$ is the set of all acceptable subobjects of $D\left(\mathrm{IM}_{J}\right)$ where each degree equals 2.

We need to show that

$$
\sum_{\substack{L \text { with each degree } 2 \\ \text { acceptable subobject of } D\left(\mathrm{IM}_{J}\right)}}(-1)^{a(L)} w_{L}=\sum_{K \text { super subobject of } \operatorname{TID}(D)}(\lambda+2)^{|V|-\operatorname{deg}_{4}(K)} 2^{\operatorname{deg}_{4}(K)}(-1)^{a(K)} .
$$

We will prove it by constructing a partition of acceptable subobjects of $D\left(\mathrm{IM}_{J}\right)$ where each vertex has degree 2 , and associating each partition class which contributes non-zero to $\operatorname{Per}\left(D\left(\mathrm{IM}_{J}\right)\right)$ with uniquelly determined super subobject of $\operatorname{TID}(D)$.

Let $L$ be an acceptable subobject of $D\left(\mathrm{IM}_{J}\right)$ where each vertex has degree 2 . Denote by $O L$ the set of all thickened arcs of type $\left(u_{i} w_{j}\right), u \neq w$, and let $I L=L-O L$. Note that if we forget the lower indices at vertices, $O L$ naturally corresponds to a set $O K$ of thickened $\operatorname{arcs}$ of $\operatorname{TID}(D)$. Note that no arc in $O K$ is a loop and $a(O L)=a(O K)$. It remains to be seen what to do with the thickened arcs of $I L$. We may consider each triple of vertices $v_{1}, v_{2}, v_{3}$ separately. Let $I L v$ denote the set of thickened $\operatorname{arcs}$ of $L$ among $v_{1}, v_{2}, v_{3}$. We distinguish four cases.

- $I L v$ consists of all three loops or the loop at $v_{1}$ and the $\operatorname{arcs}\left(v_{2}, v_{3}\right),\left(v_{3}, v_{2}\right)$. Let $C_{0}$ be the class of all $L$ which in at least one triple $v_{1}, v_{2}, v_{3}$ behave in this way. Note that the total contribution of $C_{0}$ to $\operatorname{Per}\left(D\left(\mathrm{IM}_{J}\right)\right)$ is 0 , and so we may assume that this case never happens (it corresponds to single loop at $v$ in $\operatorname{TID}(D)$ which is not allowed for supersubobjects).

- $I L v$ consists of loop at $v_{1}$ or loop at $v_{2}$ (but not both), and loop at $v_{3}$. Then let $I K v=\emptyset$. Hence $v$ will have degree 2 in $K$ and contribute $(\lambda+2)$ to $J(\operatorname{LID}(D))$, which exactly equals to the contribution of $I L v$ to $\operatorname{Per}\left(D\left(\mathrm{IM}_{J}\right)\right)$.

- $I L v$ consists of $\left\{\left(v_{2}, v_{3}\right),\left(v_{3}, v_{2}\right)\right\}$ or $\left\{\left(v_{3}, v_{1}\right),\left(v_{2}, v_{3}\right)\right\}$. Then $v$ has degree 2 in $O K$ and the edge of $O K$ incident and thickened at $v$ is entering $v$. In this case let $I K v$ consist of the loop at $v$. Note that the total contribution of $I L v$ to $\operatorname{Per}\left(D\left(\operatorname{IM}_{J}\right)\right)$ is $-(\lambda+2)+\lambda=-2$ and $2(-1)$ is also the contribution of $I K v$ to $J(\operatorname{LID}(D))$.

- $I L v=\emptyset$. Then $v$ has degree 4 in $O K$ and we let $I K v=\emptyset$. In this case each $v_{i}, i=1,2$ is incident with one arc of $L$ not thickened at $v_{i}$. Let $L^{\prime}$ be obtained from $L$ by exchanging the incidence of non-thickened arcs between $v_{1}$ and $v_{2}$. The total contribution of $I L v$ and $I L^{\prime} v$ to $\operatorname{Per}\left(D\left(\operatorname{IM}_{J}\right)\right)$ is 2 and 2 is also the contribution of $I K v$ to $J(\operatorname{LID}(D))$.

It is easy to check that we indeed partitioned the set of all acceptable subobjects of $D\left(\mathrm{IM}_{J}\right)$ where each vertex has degree 2 and that we exhausted all super subobjects of $\operatorname{TID}(D)$. This finishes the proof of Theorem 4.

\section{Proof of The COROLlaries}

Corollary 1.4 is immediate. For Corollary 1.5, observe that $W_{J J}=W_{J J^{(0)}}$ is given by Corollary 1.4 for $n=0$. We claim that this formula equals to Per(IM). This may be observed as follows: assume $i<j$. Associate $\operatorname{IM}(D)_{i j}$ with the $\operatorname{arc}(i, j)$ of $\operatorname{LID}(D)$ thickened at $i$ and $\operatorname{IM}(D)_{j i}$ with arc $(i, j)$ thickened at $j$. This associates, with each term of the expansion of Per(IM), an acceptable object of uncolored arcs only, with each degree equal to 2 and no loops. Denote the set of such acceptable objects by $\mathcal{K}_{1}$. It is straightforward to check that

$$
\operatorname{Per}(\mathrm{IM})=\sum_{K \in \mathcal{K}_{1}}(-1)^{a(K)} .
$$

On the other hand, $W_{J J^{(0)}}=\sum_{K \in \mathcal{K}_{2}}(-1)^{a(K)}$, where $\mathcal{K}_{2}$ is the set of all acceptable objects where each degree is 0 or 2 (loops are allowed and they contribute 2 to the degree). First observe that the contribution of the acceptable objects of $\mathcal{K}_{2}$ that contain a red arc cancels out since we can change the orientation of a red arc in such an object, and get again an object of $\mathcal{K}_{2}$, with oposite contribution. Hence assume $\mathcal{K}_{2}$ has no objects with red arcs. If $K \in \mathcal{K}_{2}$ then let $L(K)$ denote the acceptable subobject of $K$ obtained from 
$K$ by deleting all loops. If $L=L(K)$ let $V(L)$ denote the set of vertices of $L$ of non-zero degree and let $\mathcal{E}(L)=\left\{K \in \mathcal{K}_{2} ; L(K)=L\right\}$. By the binomial theorem,

$$
\sum_{K \in \mathcal{E}(L)}(-1)^{a(K)}=(-1)^{a(L)} \sum_{W \subset(V-V(L))}(-1)^{|W|}=0
$$

whenever $V(L) \neq V$. This proves the corollary.

\section{Numerical EXAmples}

6.1. Running the program. The formula of Theorem 1 for the weight system of the colored Jones function is easy to program, as is demonstrated by the source code given in the next section. To run the program, first start Mathematica [Wo] and load the JonesPermanent package of the next section (available from our web-site, too):

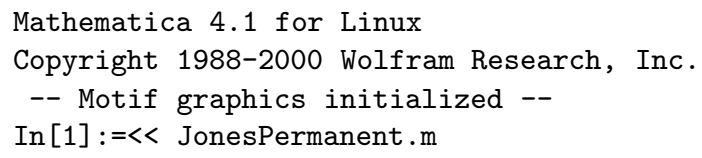

For the chord diagram CDP $[5,7,6,8,1,3,2,4]$ of Example $1.1, W_{J}$ is given by

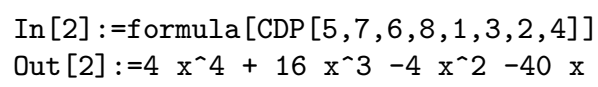

where $\lambda=x$, and the notation $\operatorname{CDP}\left[\mathrm{a}_{-} 1, \ldots, \mathrm{a}_{-}\{2 \mathrm{n}\}\right]$ for a permutation $\sigma=\left(a_{1}, \ldots, a_{2 n}\right)$ of $(1, \ldots, 2 n)$ of order two means the chord diagram of the 2-cycles of $\sigma$.

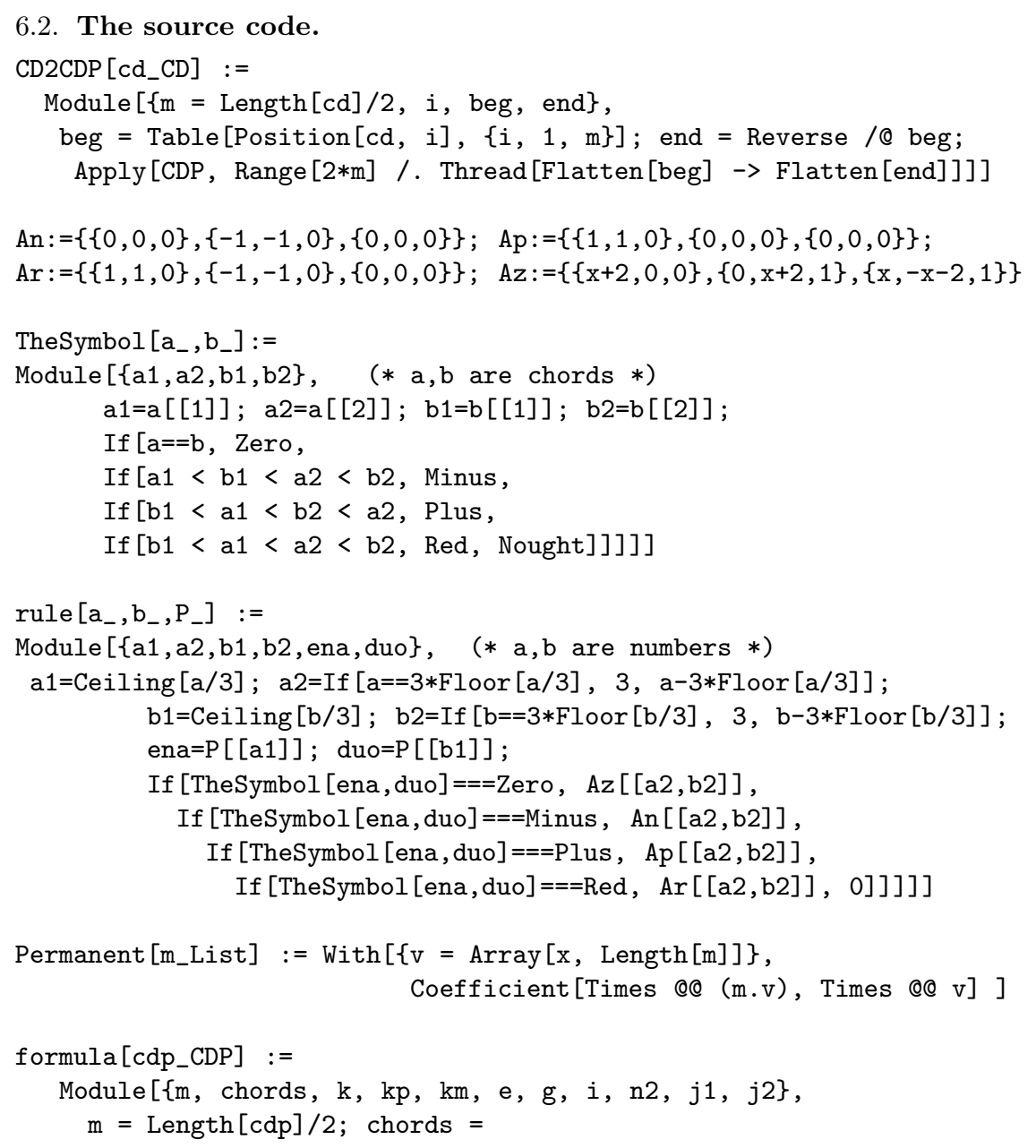


Apply [CL, Union[Sort /@ Transpose [\{Range [2*m], Apply[List, cdp] $\}]]]$;

Expand [Permanent [Table [rule $[j 1, j 2$, chords $],\{j 1,3 * m\},\{j 2,3 * m\}]]]]$

\section{REFERENCES}

[BN] D. Bar-Natan, On the Vassiliev knot invariants, Topology 34 (1995) 423-472.

[BG] and S. Garoufalidis, On the Melvin-Morton-Rozansky conjecture, Inventiones, 125 (1996) $103-133$.

[Ch] S. Chmutov, A proof of the Melvin-Morton conjecture and Feynmann diagrams, J. Knot Theory and its Rami. 7 (1998) 23-40.

[GL] A. Galluccio and M. Loebl, A Theory of Pfaffian Orientations I, II, Electronic J. of Combinatorics 6 (1999).

[GK] S. Garoufalidis and A. Kricker, A rational noncommutative invariant of boundary links, preprint 2001, math.GT/0105028.

[Hu] J. Humphreys, Introduction to Lie algebras and their representation theory, Springer-Verlag GTM 9 New York 1972.

[KSA] A. Kricker, B. Spence, I. Aitchinson, Cabling the Vassiliev invariants, J. Knot Theory and its Rami. 6 (1997) 327-358.

$[\mathrm{KM}] \quad$ B.I. Kurpita and K. Murasugi, A graphical approach to the Melvin-Morton conjecture, Topology and its Applic. 82 (1998) 297-316.

[LW] X-S. Lin and Z. Wang, Random Walk on Knot Diagrams, Colored Jones Polynomial and Ihara-Selberg Zeta Function, preprint 1998 math.GT/9812039.

[MM] P. M. Melvin and H. R. Morton, The coloured Jones function, Commun. Math. Phys. 169 (1995) 501-520.

[Ro1] L. Rozansky, A contribution of the trivial flat connection to the Jones polynomial and Witten's invariant of $3 d$ manifolds I, Commun. Math. Phys. 175 (1996) 275-296.

[Ro2] - A contribution of the trivial flat connection to the Jones polynomial and Witten's invariant of 3d Manifolds II, Commun. Math. Phys. 175 (1996) 297-318.

[Ro3] - The universal R-matrix, Bureau representation and the Melvin-Morton expansion of the colored Jones polynomial, Advances Math. 134 (1998) 1-31.

[V] A. Vaintrob, Melvin-Morton conjecture and primitive Feynman diagrams, Intern. J. Math. 8 (1997) $537-553$.

[Wo] S. Wolfram, The Mathematica Book, Cambridge University Press, 1999 and http://www.wolfram.com/.

Department of Mathematics, University of Warwick, Coventry, CV4 7AL, UK.

E-mail address: stavros@maths.warwick.ac.uk

KAM MFF UK and ITI, Charles University, Malostranske n. 25, 11800 Praha 1, Czech Republic.

E-mail address: loebl@kam.ms.mff.cuni.cz 